\title{
Entrenamiento con simuladores quirúrgicos. La Caverna de Platón, los ídolos de Bacon, la McDonaldización de la Medicina y la poca (auto) crítica
}

\author{
Surgical computer simulation training. Plato's Cavern, Bacon's Idols, \\ The McDonaldization of Medicine and poor (self) critique
}

Alberto Campos*

Palabras clave:

Cirugía general,

entrenamiento

simulado, educación quirúrgica, práctica deliberada,

simulación mejorada por tecnología, epistemología de la medicina.

Key words: General surgery, simulation training, surgical education, deliberate practice, technologyenhanced simulation, epistemology of medicine.

* Programa de Maestría y Doctorado en Ciencias Médicas, Odontológicas y de la Salud, Facultad de Medicina, UNAM.

Recibido: $15 / 06 / 2016$ Aceptado: 20/07/2016

\section{RESUMEN}

Aunque el entrenamiento con simulación mejorada por tecnología (SMT), que implica alta fidelidad, retroalimentación (feedback) háptica, y realidad virtual (RV) resulta muy atractivo, esta creencia carece de sostén empírico adecuado. Muchas de las ventajas que se le atribuyen parecen ser aparentes, percepciones e intuiciones erróneas distorsionadas por opiniones comunes poco cuidadosas. La efectividad de la SMT es difícil de evaluar, los estudios sistemáticos y los metaanálisis existentes son imperfectos y heterogéneos, de calidades muy variables e inconsistentes. La SMT provee algunas ventajas en la educación, como un ambiente controlado, posibilidades de repetición y práctica deliberada, errar y aprender de los errores, evaluación objetiva de las destrezas, y rinde mejores resultados aunque no significativos, en conocimiento, aprendizaje de habilidades y comportamientos, y un efecto global pequeño a moderado sobre el cuidado del paciente, hallazgos que podrían deberse al azar. Los simuladores no mejorados permiten un número limitado de destrezas, navegación por cámara, visión bidimensional, cortes, suturas y nudos. Los simuladores no son placebo, pero tampoco panacea; pueden ser buenos complementos en el entrenamiento médico y quirúrgico, pero no sustituyen un currículo apropiado, ni profesores dedicados ni evaluaciones rigurosas.

\section{ABSTRACT}

Although Technology-Enhanced Simulation (TES) training which implies high fidelity, haptic feedback, and virtual reality $(V R)$ is very attractive, this belief lacks adequate empirical support. Many of the advantages attributed to it seem only apparent, intuitions and erroneous perceptions distorted by common, careless views. The effectiveness of TES is difficult to assess, the existing systematic studies and meta-analysis are imperfect and heterogeneous, highly variable and of inconsistent quality. TES provides some advantages in education, such as a controlled environment, replicability and deliberate practice, to err and learn from mistakes, an objective assessment of skills, and yields better although not significant results in knowledge, learning skills and behaviors, and have a small to moderate effect on overall patient care, findings that could be due to chance. Unimproved simulators allow a limited number of skills, navigation by camera, two-dimensional view, cuts, sutures and knots. Simulators are no placebo, but no panacea; they can be good complements in medical and surgical training, but do not replace an appropriate curriculum, dedicated teachers and rigorous evaluations.

\section{INTRODUCCIÓN}

\section{Simuladores, la Caverna de Platón y los ídolos de Bacon}

$\mathrm{E}_{\mathrm{n}}^{\mathrm{n}}$ la alegoría de La Caverna, en el libro VII de La República, Platón habla de un mundo sensible, donde están los objetos que percibimos, y las imágenes o apariencias de esos objetos, que no son más que semejanzas de realidades que no podemos percibir, accesibles a la parte racional, y que se encuentran en el mundo inteligible, de las ideas. En la alegoría, Sócrates cuenta a Glaucón cómo hay en la Caverna unos prisioneros atados, obligados a mirar a una pared frente a ellos, cómo la luz 
de un fuego proyecta en esa pared las sombras de marionetas manejadas sobre un muro detrás de ellos y que no pueden ver, pues no pueden voltear, y cómo los habitantes de la caverna piensan que eso, lo que ven en el muro, es todo lo que existe. ${ }^{1}$

Así son las creencias - dice Sócrates a Glaucón- de quienes no pueden conocer más allá de las percepciones inmediatas de los sentidos. En una de las varias y muy divergentes interpretaciones de la imaginería de la caverna, Platón lleva tan lejos su antipatía hacia un estado pasivo y condescendiente, que presenta ese estado de ignorancia como inferior, un estado en el que sólo se ven las sombras de las marionetas, pero no las marionetas mismas. Las creencias comunes no van más allá de la vista de sombras, ilusiones en primer grado, y ésa es la gran diferencia entre quienes entienden y quienes no, porque no habitan el mismo mundo cognitivo. ${ }^{2}$ Los habitantes de la caverna asumen irreflexivamente opiniones y creencias de segunda mano; son ignorantes de su ignorancia.

¿Pero, por qué citar la Caverna de Platón? ¿Por qué examinar una metáfora griega sobre la naturaleza del conocimiento en un artículo quirúrgico? Porque frecuentemente nosotros, como ellos, creemos que sabemos, pero sólo unos pocos pueden darse cuenta de que las sombras son sólo sombras de marionetas. La alegoría de La Caverna bien puede aplicarse a lo que un simulador quirúrgico proyecta sobre la realidad, respecto del cuerpo humano y de los fenómenos que se suceden a lo largo de un acto quirúrgico. Al contrario del criterio común, un simulador es sólo una marioneta, en el sentido más estricto; ciertamente una marioneta con alto grado de complejidad tecnológica, pero nada más, y las imágenes que proyecta son sombras, algunas muy sofisticadas y sutiles, pero nada más. El simulador hace lo que el operador comanda, pero nada más. Por el contrario, en el paciente quirúrgico, en el trans y posoperatorio, cualquier cosa puede suceder.

Analicemos pues otra metáfora sobre la relación entre las apariencias de un simulador y el paciente real. Para Francis Bacon la inteligencia es un instrumento eficaz para entender la naturaleza, pero para ello es necesario librarse de prejuicios y acudir a la experiencia directa. En Valerius Terminus. De la interpretación de la
Naturaleza (1603), expone una primera clasificación de los ídolos (idola), falsas apariencias o falacias que acosan la mente, "errores nativos e inherentes en la mente del hombre, que han coloreado y corrompido todas sus nociones e impresiones". ${ }^{3}$

Años más tarde, Bacon escribe el Novum Organum (1620), precisamente un compromiso fuerte con los nuevos instrumentos técnicos y la creciente variedad de experimentos que éstos permitirían llevar a cabo con la naturaleza, para modelar lógicamente el surgimiento de la ciencia experimental, para investigar sus premisas fundamentales con base en la observación de la evidencia del mundo natural, no de imágenes de éste. Ahí describe con detalle los Ídolos de la Tribu, "fundados en cada tribu o raza de la humanidad" errores en la percepción misma, pues "tanto los sentidos como la mente tienen como referencia el hombre, no el universo. $[E] l$ entendimiento humano es como un espejo poco uniforme que [...] lo distorsiona y corrompe". Los Ídolos de la Cueva, referencia a la Caverna de Platón, "un tipo de caverna individual que fragmenta y distorsiona la luz de la naturaleza - dice, pues- el espíritu humano es una cosa variable, bastante irregular, casi por azar". Los Ídolos del Mercado, que refieren al uso del lenguaje cotidiano entre la gente común, pues "las palabras comunes violentan el entendimiento y confunden todo, y traicionan a los hombres hacia incontables ficciones y disputas vacías". Finalmente, los Ídolos del Teatro, "ilusiones de los varios dogmas de diferentes filosofías que han hecho su morada en las mentes de los hombres [...] principios y axiomas de las ciencias que se han fortalecido de la tradición, la creencia y la inercia." "El entendimiento - continúa- supone un orden y regularidad mayor de la que encuentra [...] inventa paralelos y correspondencias y conexiones inexistentes [y] esta vanidad prevalece no sólo en los dogmas sino también en nociones sencillas." ${ }^{4}$ Pero al contrario de Platón, a quien critica junto con Aristóteles y otros filósofos de la antigüedad puramente especulativos, Bacon no cree que la verdad se encuentre en un mundo más allá de la experiencia sensible, sino precisamente en este mundo, sólo que es preciso obligar a la naturaleza a que se nos muestre. 
De tal suerte, pueden verse los simuladores quirúrgicos como ídolos baconianos en vez de instrumentos cognitivos cuando se les atribuyen ingenuamente más cualidades que las que tienen, cuando refieren a un universo virtual y no al individuo concreto, cuando se da por sentado, o se pretende, que gracias a ellos el entrenamiento del cirujano está garantizado.

En un trabajo previo he analizado algunos argumentos con los que se justifica el uso de simuladores, pero los simuladores quirúrgicos producen ilusiones hápticas que, por muy reales que parezcan, no pasan de ser metáforas táctiles, muy sofisticadas marionetas programadas del cuerpo humano a semejanza del cual están hechos, sin ser el propio objeto. ${ }^{5}$ Estas metáforas, imperfectas por incompletas, en tanto ilusiones cavernarias o ídolos baconianos, y a pesar de la muy alta resolución de imagen que puedan desplegar, no permiten conocer el cuerpo.

Propongo entonces, en este ensayo, analizar algunos problemas derivados del uso de simuladores, si la evaluación de ese uso ha sido adecuada y si hay evidencia de que realmente contribuyan, más allá de la adquisición de destrezas manuales, a la formación de cirujanos.

\section{Simuladores y la McDonaldización de la Medicina}

Parte de un proceso de racionalización de recursos es la sustitución de valores y tradiciones de una sociedad, tenidos como poco eficientes, por incentivadores racionales para aumentar la capacidad de producción. Las burocracias son ejemplo de ese proceso. Pero tal racionalización no sólo no garantiza el progreso; produce un falso efecto de éste y una deshumanización en muy disímbolos terrenos interconectados en una trama psicológica social, invisible por insospechada. El modelo, emergente en los años ochenta y bien afianzado ya, es el restaurante de comida rápida, McDonald's; con sus cuatro principios básicos, eficiencia, calculabilidad, predictibilidad y control de los procesos, es el arquetipo. ¿Es eficiente? Sí. ¿Produce la mejor comida? No. Distintas actividades sociales, la educación, los deportes, las políticas públicas, han adaptado esos principios a su manera de proceder; en todas ellas se ha perdido calidad. ${ }^{6}$
Todos esos fenómenos sociales participan de lo que Max Weber llamó el proceso de racionalización, cuya consecuencia final es el control de los humanos por tecnología no humana. ${ }^{7}$

$\mathrm{Ni}$ la Medicina ni su enseñanza, queriendo ser eficientes desde la relación costo/beneficio, escapan al proceso. No confundamos eficiencia con eficacia, no es lo mismo; de acuerdo con el Diccionario de la lengua española (DLE, 23a ed., 2014), eficiencia es la "capacidad de disponer de alguien o de algo para conseguir un efecto determinado", eficacia es la "capacidad de lograr el efecto que se desea o se espera." Entonces, disponer de algo no garantiza que se logre nada. Si no se tiene la capacidad, el medio no garantiza el fin.

De nuevo, ni la racionalización ni los simuladores pueden sustituir el entrenamiento supervisado de campo; no pueden suplir lo que los exámenes y los controles anuales de calidad de la enseñanza no hacen. "Lo que un tutor de cirugía no da simulador no otorga".

Por el contrario, los cuatro principios básicos de la McDonaldización de la Medicina pueden conllevar efectos adversos; por ejemplo, los expedientes electrónicos controlan las interacciones entre médicos y pacientes especificando qué preguntas deben hacerse y qué tareas deben completarse. Estas tecnologías pueden reducir a los trabajadores de comida rápida y a los médicos en autómatas. A pesar de que la eficiencia, calculabilidad, predictibilidad y control que el modelo promueve sean benéficos, el mensaje parece ser "estandariza, no pienses." Con una diferencia, que mientras el empleado de un fast-food no necesita pensar mucho, al médico le es más que indispensable, esencial.

Los simuladores quirúrgicos son otro ejemplo de racionalización anómala. La tendencia hacia una eficiencia siempre creciente del sistema puede formar cirujanos hábiles de pinzas pero inexpertos; cuando el valor de la enseñanza pasa de la calidad a la cantidad de alumnos, cuando la productividad se mide en números, cuando se da mayor peso a la destreza, en tanto "habilidad, arte, primor o propiedad con que se hace algo" (DLE) que a la aptitud, en tanto "capacidad para operar competentemente, suficiencia o idoneidad para ejercer un empleo" (DLE), cuando se da mayor peso 
al adiestramiento mediante la tecnología que al conocimiento de la casi olvidada enseñanza clínica, personal, in situ, cuando los residentes pasan más tiempo frente a la computadora o la tableta que frente al paciente..$^{8,9}$

Como resultado de la racionalización, los juicios subjetivos y autónomos de los médicos empiezan a ser reemplazados por resultados objetivos y cuantitativos que surgen de tecnologías varias, por ejemplo, programas por computadora que ciertamente ayudan al médico y que son más replicables y predecibles, pero que ejercen mayor control externo sobre éste y lo hacen rutinario, $y$ al hacerlo, erosionan su cuerpo de conocimientos sistemáticos. ${ }^{10}$ En otras palabras, estos cambios promueven una racionalidad mecánica, descuidada, pro forma, que se está dando en universidades y hospitales, públicos y privados, que tiene por objeto mejorar el proceso y por resultado lo contrario.

\section{DISCUSIÓN}

\section{Las limitaciones y la poca (auto) crítica}

Tradicionalmente, el entrenamiento quirúrgico se ha basado en un periodo más o menos largo de aprendizaje, con la práctica en pacientes, supervisada por tutores. Pero nuevas preocupaciones y directrices respecto de tiempos más cortos de entrenamiento y de admisión de pacientes, que emanan de la racionalización de los procesos, tienden a reducir el tiempo de aprendizaje, a cambiar el enfoque del individuo al equipo, por ejemplo, en anestesia; en fin, a rediseñar la fuerza de trabajo y las funciones profesionales, de modo que los procedimientos invasivos son llevados a cabo cada vez más por internistas, radiólogos y cardiólogos intervencionistas y otros. ${ }^{11,12}$

Por el lado contrario, el desarrollo de experiencia exige una práctica deliberada y sostenida, y el número de horas invertido en la práctica deliberada correlaciona con altos niveles de destreza. El concepto no es nuevo, un pianista lo sabe. El problema epistemológico es entender las fortalezas y limitaciones de los simuladores, determinar su eficacia e identificar muy claramente su papel en la educación de los cirujanos. La estrategia debe incluir también estandarización de la documentación de habilidades y progresos obtenidos. ${ }^{13}$
Por una parte, es una preocupación legítima el que los practicantes adquieran experiencia con pacientes, y por otra, las nuevas técnicas, como cirugía de invasión mínima y los procedimientos asistidos por imagen, requieren familiarización con la tecnología y el desarrollo de nuevas habilidades. Por otra parte, la simulación mejorada por tecnología (SMT) es extremadamente cara, pero los simuladores de menor fidelidad o aquellos para tareas sencillas como orientación espacial o suturas, de menor costo, son suficientes para esas funciones pero insuficientes para formar un cirujano. De tal suerte, el argumento por controvertir es el diseño educativo y la adecuación de los simuladores para esos propósitos, y no la complejidad de la simulación per se.

Hay también una tensión entre el diseño y la evaluación de un simulador; para el desarrollador representa el fin del desarrollo, para el evaluador, el principio de un proceso largo que, además, debe ser paralelo al currículum. ${ }^{14} \mathrm{En}$ pregrado, los simuladores sirven para enseñar destrezas manuales; en la especialidad, para contrastar los entornos simulados con pacientes reales, y es ahí donde son más útiles como complemento de un entrenamiento siempre supervisado por profesores hospitalarios.

Sin una adecuada (auto) crítica, una evaluación de la validez de su contenido y de su fidelidad, y de la correcta transferencia de habilidades, no se sabrá si las aprendidas en un simulador pueden aplicarse exitosamente en la práctica clínica. Los simuladores podrían entonces, potencialmente, dañar pacientes si contribuyen a un falso sentido de confianza. La prueba final por evaluar es el acto quirúrgico.

Hay poca investigación sobre la efectividad de la tecnología de simulación en la educación médica, inconsistente y muy variada en rigor metodológico y enfoque. Ya Miller había propuesto una tipología de cuatro niveles para evaluar a un aprendiz en medicina, (a) sabe (conocimiento), recuerda hechos, principios, teorías; (b) sabe cómo (competencias), habilidad para resolver problemas, ser funcionalmente adecuado, competente; (c) muestra cómo (desempeño), demuestra habilidades en un entorno controlado; (d) hace (acción), comportamiento en la práctica real. Los tres primeros pueden ser simulados, el último es 
difícil de evaluar. Generalmente no se reconoce la falta de estandarización en la evaluación de esta última competencia, y por eso mismo, se hace más con base en impresiones clínicas que mediante una acumulación sistemática de información confiable. ${ }^{15}$

Otros han encontrado problemas similares, confusores como la aleatorización, el cegado y la contaminación del brazo de intervención, deficiente estandarización, métricas no comparables, malas definiciones de variables operativas, falta de rigor cuantitativo, mayor número de estudios descriptivos que de evaluación de resultados, muestras muy pequeñas no validables, falta de datos publicados de alta calidad, así como carencia de estudios longitudinales bien diseñados para evaluarlos. ${ }^{14,16}$

En un metaanálisis de la historia de los simuladores durante 34 años, el $80 \%$ de los resultados encontrados por Issenberg et al., fue "equívoco, en el mejor de los casos" y "sólo $20 \%$ de las publicaciones revisadas reportaron resultados claros o probablemente verdaderos." Dadas las limitaciones de la evidencia publicada, Issenberg et al. incluso descartan un metaanálisis formal y refieren que, a la luz de esas fallas en el diseño de los estudios, es imposible una síntesis cuantitativa de la evidencia. ${ }^{17}$

Análogamente, en otro metaanálisis, en una búsqueda sistemática de estudios cuantitativos en un periodo de 10 años, de 2, 019 referencias localizadas de reportes de estudios cuantitativos exclusivamente en maniquíes, Cant y Cooper incluyeron apenas 12 que tenían criterios Comunes; 7 de esos 12 incluyeron cuando menos una medida validada de evaluación, en los restantes la confiabilidad no era clara. Encontraron que frecuentemente se usó una escala de auto confianza como indicador de habilidad clínica. Sólo 6 estudios de los que compararon simulación con grupo control fueron capaces de mostrar ganancia adicional en conocimiento, pensamiento crítico y confianza percibida. Sin embargo, la falta de comparación con otras estrategias educativas como seminarios clínicos o estudios de caso, de tomarse en cuenta, habrían podido reducir aún más el efecto de la comparación. Además, la variabilidad de la evaluación de los resultados tenía un sesgo asociado, el de compararlos inmediatamente después de terminado el curso y evaluar la retención a una semana y un mes después de la intervención, con los sesgos agregados de que, las evaluaciones fueron indirectas y auto reportadas en términos de las percepciones de los entrenados sobre su propio pensamiento crítico, y de que se usaron escalas de auto confianza para la toma de decisiones, este solipsismo en los juicios limita la generalizabilidad de las revisiones. ${ }^{18}$

Aún un metaanálisis cuantitativo que comparó la evidencia entre educación médica basada en simulación más práctica deliberada $(E M B S+P D)$ vs educación tradicional en un periodo de 20 años, en el que, de 3,742 artículos identificados sólo 14 cumplieron con los criterios de inclusión, concluye que la educación médica con base en simuladores es "superior a la educación médica clínica tradicional para alcanzar metas específicas de adquisición de habilidades clínicas" para competencias como Advanced Cardiac Life Support, técnicas quirúrgicas laparoscópicas, inserción de catéter venoso central, auscultación cardiaca y toracocentesis, pero sólo 6 estudios demostraron mejora en habilidades quirúrgicas laparoscópicas como colecistectomía, manejo de instrumentos, navegación con la cámara y suturas de tejidos vivos. Aun así, el metaanálisis aborda la adquisición de habilidades procedimentales, pero "no cubre la adquisición de otras habilidades clínicas, tales como evaluación bajo presión, toma de decisiones médicas, y conciencia de la situación", y los autores, McGaghie et al., admiten que no se sabe si el modelo EMBS+PD es adecuado para estas últimas habilidades, lo que justifica hacer investigación al respecto. ${ }^{19}$

Los simuladores aportan ventajas a la enseñanza en tanto permiten desarrollar habilidades, permiten errar sin consecuencias, la mecanización de movimientos y una cantidad casi ilimitada de repeticiones, ventajas que ciertamente no podrían lograrse ni en cadáveres ni en pacientes. Pero tienen grandes desventajas que es necesario ponderar, el enfoque en las tareas puramente técnicas puede hacer que los cirujanos pierdan la visión panorámica de la cirugía; por ejemplo, adquirir una visión en túnel y confiar en que, si no se ve la hemorragia en pantalla, no existe, como es el caso de movimientos descuidados o poco delicados de 
los trócares. Puesto que la SMT (TechnologyEnhanced Simulation, TES) que incluye simulación de alta fidelidad, con retroalimentación (feedback) háptica, o de realidad virtual, no está disponible en todas partes, por causa de su costo, un programa de entrenamiento en simuladores no mejorados permitirá sólo un número muy limitado de destrezas, apenas la navegación por cámara, la visión bidimensional, algunos cortes suturas y nudos. ${ }^{20}$

Aún así, la efectividad de la SMT es incierta, a la luz de un metaanálisis reciente de Cook et al., de 609 estudios elegibles (a partir de una revisión de 10,903 artículos) que involucraron 35,226 aprendices, en el que preguntaron específicamente en qué medida se asocian la SMT con mejores resultados, comparando con grupos sin intervención y cómo varían los resultados según el diseño de la simulación. Encontraron que la SMT tuvo, mejores resultados de conocimiento, aprendizaje de habilidades y comportamientos, aunque un efecto global pequeño a moderado sobre el cuidado del paciente, concluyendo que los hallazgos podrían deberse al azar en evaluaciones con importantes inconsistencias, como grupos heterogéneos de aprendices, diseño de la instrucción, calidad de la investigación, muestras pequeñas. Con todo, concluyen que la SMT aporta modestas ventajas. La cuestión importante que plantean es cuándo y cómo usar una simulación cognitivamente más efectiva y eficiente en costo. ${ }^{21}$

La disponibilidad, el consumo y el abasto son problemas no menores, más importantes que instalar el centro de simulación, puesto que garantizan la continuidad del programa. Dado su muy alto costo, sobre todo cuando se trata de simuladores de alta fidelidad y realidad virtual, parecería una estrategia segura equipar un centro con un pequeño conjunto de simuladores básicos. Pero -reiteración deliberada- por lo básico, sólo sirven para adquirir destrezas básicas. Centros así servirían para unos pocos ejercicios de coordinación en un cubo si no hay un compromiso continuado de quienes manejan los presupuestos en universidades y hospitales. La oferta de poca diversidad tiene como consecuencia la poca demanda, salas de simulación que se usan poco, construidas para un propósito determinado pero no concluidas por falta de presupuesto o causas administrativas; que terminan siendo entonces simulaciones, elefantes blancos.

No se puede pretender tener un centro de simulación si lo anterior no se asume, o si, una vez abierto, por las justificaciones macroeconómicas y financieras que se quieran, se maneja con políticas modestas de presupuestos modestos, con tal de mantener viva la referencia de que se tiene un simulador. Abrir un centro de simulación requiere de un compromiso académico fuerte, no sólo unos cuantos pasos. Por otra parte, los residentes debieran ser informados, antes de elegir, sobre las instalaciones de los hospitales donde pretenden entrenarse, así como de la idoneidad del cuerpo de profesores. ${ }^{22}$

\section{Validación de los simuladores 0 validación de la simulación}

Ciertamente son necesarias estrategias de validación y estandarización del uso de simuladores a escala nacional y por las diferentes universidades y hospitales que los proveen y abogan por ellos. Eso requerirá un enorme esfuerzo de consenso para proporcionar a los estudiantes de pregrado y a los residentes un currículo adecuado, validado en contextos apropiados y mediante trabajo en equipo, posiblemente por medio de intercambios académicos, lo que permitiría, además de no duplicar recursos, la optimización y la variedad de los ya existentes. Un trabajo de consenso. Un esfuerzo más de universidades que de asociaciones independientes que, por otra parte, también han invertido en simuladores y desearían legítimamente amortizar sus gastos.

En fin, otro punto no menor, un conjunto de tutores y expertos en SMT, cirujanos expresamente entrenados, no solamente ingenieros o técnicos, ciertamente no pasantes con nula experiencia quirúrgica, aunque puedan tener mucha en video juegos; aprendices que entrenan aprendices. Para entrenar se requieren profesionales que acrediten ese entrenamiento, certificados, reconocidos por las varias universidades de tal sistema de cooperación. Para ello hay que crear las condiciones, facilitar el trabajo de los equipos, crear oportunidades para que los expertos (reitero, cirujanos con experiencia) se entrenen primero en esas tareas complejas 
de simulación, para luego entrenar aprendices. De lo contrario, el entrenamiento mediante simuladores no pasará de ser una simulación.

\section{CONCLUSIONES}

Me gustaría iniciar el cierre con una vuelta al Nuevo Órganon de Bacon, que toma su título del Órganon (Instrumento) de Aristóteles, un instrumento para el pensamiento racional. El Nuevo Órganon propone un sistema para la búsqueda del conocimiento en la ciencia. Mientras el sistema inferencial aristotélico se basa en silogismos para derivar conclusiones lógicamente consistentes con las premisas, el sistema baconiano fue diseñado para investigar las premisas fundamentales mismas. Donde Aristóteles propuso certezas con base en enunciados incontrovertibles pero no demostrados, Bacon propuso inferencias inductivas, con base en un retorno a la evidencia. Su crítica, tanto a la tradición sin cuestionar como a la novedad deslumbrante, portentosa, es muy clara:

"Algunas mentes se dedican a la admiración de la antigüedad, otras al amor de la novedad, y pocas tienen el temperamento de mantenerse en la media sin criticar los verdaderos logros de los antiguos ni despreciar las contribuciones reales de los modernos. Esto es una gran pérdida para las ciencias y para la filosofía, pues éstos no son juicios sino entusiasmo por la antigüedad o la modernidad. [...] Es preciso rechazar estos entusiasmos y asegurarnos de que el entendimiento no se desvíe hacia la conformidad con ellos."23 (Cursivas añadidas.)

La cita extensa viene a colación como una reflexión frente a los ídolos, resplandores y sombras de marionetas que también pululan inadvertidos en la cirugía, una reflexión frente al lugar común y vulgar descrito por la expresión "el vertiginoso avance de la ciencia". A quien la ciencia produzca vértigo, que no se acerque. Además de eso, la expresión no dice nada.

Respecto de quienes se deslumbren por los simuladores y los propongan sin una adecuada (auto) crítica, tengamos en cuenta que el brillo en el cenit académico puede ser entretenido y deslumbrar bienaventurados, pero es ocioso. No produce nada. Y los simuladores quirúrgicos distan aún de estar en el cenit. Son herramientas útiles que facilitan el desarrollo de compe- tencias psicomotoras, un proceso con base en la práctica deliberada; pero la simple repetición de tareas no basta para formar cirujanos competentes. Persiste el hiato epistémico antes mencionado, cognitivo, háptico, tecnológico. ${ }^{5}$ Son retos aún no resueltos. Sigue faltando una métrica de la eficiencia de los contenidos de los simuladores, que sea basada en evidencia, mediante estudios, si no prospectivos, al menos retrospectivos pero adecuadamente estandarizados, que sean comparables. El problema de transferencia de habilidades de la simulación a la vida real aún permanece sin respuesta.

Existe además el peligro de que quienes tienen capacidad y recursos para desarrollar simuladores y venderlos mantengan agendas diferentes de las agendas universitarias y hospitalarias, y de que éstas se deslumbren con la novedad; existe el peligro de que permanezca el hiato entre las habilidades aprendidas en simulador y las habilidades de la clínica quirúrgica sin una manera clara de tender un puente mediante un currículo adecuado; existe el peligro de que los aprendices se hagan expertos en simulaciones y no en los contextos clínicos que éstas intentan reflejar. ${ }^{12,14}$

La simulación no sustituye a un entrenamiento curricular bien diseñado, con suficiente cobertura de contenidos y de horas de práctica tuteladas; los simuladores son sistemas autónomos que tienen valor sólo en el contexto de un currículo educativo completo, que debe ser el centro del programa. ${ }^{24}$ Su principal bondad es que permite la práctica en un entorno controlado, seguro e indulgente para el aprendiz, quien puede enfocarse en procedimientos específicos y practicarlos tan frecuentemente como sea necesario, con un menor costo en términos de iatrogenia. ${ }^{14,16}$ Pero, sobre todo, la simulación bien puede ser el complemento de un entrenamiento con un currículo teórico y empírico bien planeado cuyo contenido y duración, a la luz de la tasa de fracaso en el examen de certificación del Consejo Mexicano de Cirugía General de "alrededor del 40\%", 25 también convendría revisar. Las causas de ese fracaso están en otra parte, no dependen ni pueden ser paliadas por simuladores.

Concluyo con una ironía frente al exceso de racionalización posmoderna. Ciertamente, la eficiencia, calculabilidad, predictibilidad y el 
control mediante estandarización de algunas prácticas son benéficos; el problema se da a partir de una simplificación de la realidad, de un exceso de confianza en objetivos ingenuos con base en tecnologías que, sin vigilancia, conducen a ajustar técnicas con guías - nada menos, pero nada más - conducen a la inercia intelectual y a la poca (auto) crítica, a la formación de McSurgeons.

\section{REFERENCIAS}

1. Platón. Libro VII (514a-520a). En: The Republic. (With an English Translation by Paul Shorey) Vol II. Cambridge, MA, Harvard University Press; Londres, William Heinemann Ltd; 1935 (Reimp. 1942). p. 118-233.

2. Annas, Julia. Understanding and the Good: Sun, Line, and Cave. En: An Introduction to Plato's Republic. Nueva York, NY: Oxford University Press; 1981. p. 242-271.

3. Bacon, Francis. Valerius Terminus. Of the Interpretation of Nature. Cap. 16. Of the Internal and Profound Errors and Superstitions in the Nature of the Mind, and of the Four Sorts of Idols or Fictions Which Offer Themselves to the Understanding in the Inquisition of Knowledge. Adelaida: The University of Adelaide Library; 2014.

4. Bacon, Francis. The New Organon. (Ed. Jardine L \& Silvethorne M.) Libro I, Fragm. xxxix - xlv. Cambridge: Cambridge University Press; 2003. p. 39-42.

5. Campos A. Entrenamiento con simuladores quirúrgicos. ¿̇nstrumentos cognitivos o metáforas táctiles? Cirujano General. 2015; 37: 109-111.

6. Dorsey ER, Ritzer G. The McDonaldization of Medicine. JAMA Neurol. 2016; 73: 15-16.

7. Ritzer G. La McDonaldización de la sociedad. Un análisis de la racionalización en la vida cotidiana. Barcelona: Ariel; 1996.

8. Block L, Habicht R, Wu AW, Desai SV, Wang K, Silva KN, et al. In the wake of the 2003 and 2011 duty hours regulations, how do internal medicine interns spend their time? J Gen Intern Med. 2013; 28: 1042-1047.

9. Mamykina L, Vawdrey DK, Hripcsak G. How Do Residents Spend Their Shift Time? A Time and Motion Study with a Particular Focus on the Use of Computers. Acad Med. 2016; 91: 827-832.

10. Ritzer G, Walczack D. Rationalization and the Deprofessionalization of Physicians. Soc Forces. 1988; 67: 1-22.

11. Evgeniou E, Loizou P. Simulation-based surgical education. ANZ J Surg. 2013; 83: 619-623.
12. Kneebone RL, Nestel D, Vincent C, Darzi A. Complexity, risk and simulation in learning procedural skills. Med Educ. 2007; 41: 808-814.

13. Gould D. Using simulation for interventional radiology training. Br J Radiol. 2010; 83: 546-553.

14. Kneebone R. Simulation in surgical training: educational issues and practical implications. Med Educ. 2003; 37: 267-277.

15. Miller GE. The assessment of clinical skills/competence/ performance. Acad Med. 1990; 65: S63-S67.

16. Lurie SJ. Raising the passing grade for studies of medical education. JAMA. 2003; 290: 1210-1212.

17. Issenberg SB, McGaghie WC, Petrusa ER, Gordon DL, Scalese RL. Features and uses of high-fidelity medical simulations that lead to effective learning: a BEME systematic review. Med Teach. 2005; 27: 10-28.

18. Cant RP, Cooper SJ. Simulation-based learning in nurse education: systematic review. J Adv Nurs. 2010; 66: 3-15.

19. McGaghie WC, Issenberg SB, Cohen ER, Barsuk JH, Wayne DB. Does Simulation-Based Medical Education with Deliberate Practice Yield Better Results than Traditional Clinical Education? A Meta-Analytic Comparative Review of the Evidence. Acad Med. 2011; 86: 706-711.

20. Agha RA, Fowler AJ. The Role and Validity of Surgical Simulation. Int Surg. 2015; 100: 350-357.

21. Cook DA, Hatala R, Brydges R, Zendejas B, Szostek $\mathrm{JH}$, Wamg AT, et al. Technology-Enhanced Simulation for Health Professions Education. A Systematic Review and Meta-analysis. JAMA. 2011; 306: 978-988.

22. Milburn JA, Khera G, Hornby ST, Malone PS, Fitzgerald JE. Introduction, availability and role of simulation in surgical education and training: review of current evidence and recommendations from the Association of Surgeons in Training. Int J Surg. 2012; 10: 393-398.

23. Bacon, Francis. The New Organon. (Ed. Jardine L \& Silvethorne M.) Libro I, Fragm. Ivi Cambridge: Cambridge University Press; 2003. p. 47.

24. Satava RM. Surgical Education and Surgical Simulation. World J Surg. 2001; 25: 1484-1489.

25. Cervantes J. Surgical Education in Mexico. World J Surg. 2010; 34: 875-876.

Correspondencia:

Alberto Campos

Unidad de Posgrado. Edificio A, primer piso. Circuito de Posgrados, Ciudad Universitaria, 04510, Ciudad de México.

E-mail: alberto_campos@hotmail.com https://orcid.org/0000-0001-5811-1908 